\title{
Erratum to: Transitional cell carcinoma in urachal cyst
}

Himanshu C. Soni, Sapna Marda, Kirti G. Goswami, Harshna Vadvala, Keyur Parekh, Himanshu Vadodaria

Department of Radio-diagnosis, Gujarat Cancer \& Research Institute, Asarwa, Ahmedabad 380016, Gujarat, India

Erratum to: Abdom Imaging (2010) 35:764-766 DOI 10.1007/s00261-009-9576-X

One of the co-author Keyur Parekh's family name was erroneously submitted and published with a typo, as Parikh, it should be Parekh.

The online version of the original article can be found under doi: 10.1007/s00261-009-9576-x. 